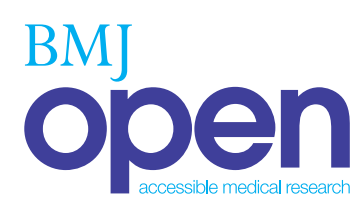

\title{
Assessment of whole body MRI and sestamibi technetium-99m bone marrow scan in prediction of multiple myeloma disease progression and outcome: a prospective comparative study
}

To cite: Khalafallah $A A$, Snarski A, Heng $R$, et al. Assessment of whole body MRI and sestamibi technetium-99m bone marrow scan in prediction of multiple myeloma disease progression and outcome: a prospective comparative study. BMJ Open 2013;3: e002025. doi:10.1136/ bmjopen-2012-002025

- Prepublication history for this paper are available online. To view these files please visit the journal online (http://dx.doi.org/10.1136/ bmjopen-2012-002025).

Received 28 August 2012 Revised 30 November 2012 Accepted 03 December 2012

This final article is available for use under the terms of the Creative Commons Attribution Non-Commercial 2.0 Licence; see http://bmjopen.bmj.com

For numbered affiliations see end of article.

\section{Correspondence to} Dr Alhossain A Khalafallah; khalafallah@dhhs.tas.gov.au

\section{ABSTRACT}

Objectives: This study aims primarily to determine whether whole body MRI (WB-MRI) and Sestamibi Technetium-99m-bone marrow (MIBI) scans in the same patients produce the same estimate of disease load and location, and secondly, to study possible association between the bone disease detected by these scans and the effect on disease outcome and survival. Bone disease occurs in about $90 \%$ of multiple myeloma (MM) patients. There are no data comparing the new diagnostic modalities with WB-MRI and MIBI in MM.

Design: A prospective comparative study between WB-MRI and MIBI scans in assessing bone disease and outcome of MM.

Participants and methods: Sixty-two consecutive patients with confirmed MM underwent simultaneous WB-MRI (both axial T1 and turbo spin echo short tau inversion recovery (STIR)) and MIBI scans at a single institution from January 2010 to January 2011, and their survival status was determined in January 2012. The median age was 62 years (range 37-88) with a male-to-female ratio of $33: 29$.

Results: In vertebrae and long bones, MRI scan detected more disease compared with MIBI scan $(p<0.001)$ but there was less difference in the skull $(p=0.09)$. In the ribcage, the MIBI scan detected more lytic lesions of the ribs compared with MRI scan $(p<0.001)$. Thirteen of the 62 patients died during the 24-month follow-up. Increased disease detected in all bones by both scans was associated with increased mortality risk (MIBI $p=0.001$; MRI-STIR $p=0.044$; but not MRI-T1 $p=0.44$ ). In all combined bone groups, the mean MIBI scan results provided a better prediction of mortality than MRI scan over the follow-up period (MRI-T1 vs MIBI $p=0.019$; MRI-STIR vs MIBI $\mathrm{p}=0.047$ ).

Conclusions: Although WB-MRI detected more MM bone disease, MIBI scan predicted overall disease outcome and mortality better than MRI scan. Further studies to define optimum use of these imaging techniques are warranted.

\section{ARTICLE SUMMARY}

Article focus

- Bone disease occurs in about $90 \%$ of multiple myeloma (MM) patients.

- Among the four available radionuclear imaging techniques, there are no data comparing wholebody MRI (WB-MRI) and Sestamibi Technetium99m-MIBI bone marrow (MIBI) scan in assessing bone disease in MM.

- Therefore, there is a growing interest in the role of MIBI scanning in conjunction with WB-MRI in determining the extent of myeloma bone disease and the prediction of disease outcome.

Key messages

- WB-MRI scan identifies more myeloma bone disease than the MIBI scan with more sensitivity in detecting myelomatous deposits in the spine and to a lesser extent, in the pelvic and long bones. However, in the ribcage, MIBI scan detected more disease compared to MRI scan $(p<0.001)$.

- A novel finding shows that MIBI scan obtains an image of all important bone compartments in the body in one single examination, and is less time consuming and more comfortable for the patient than MRI.

- MIBI scan was able to predict overall disease outcome and mortality better than MRI scan.

Trial registration number: The study was registered prospectively in the Australian and New Zealand Clinical Trials Registry at http://www.ANZCTR.org.au under No: ACTRN12609000761268.

\section{INTRODUCTION}

Multiple myeloma (MM) is a malignant neoplasm of plasma cells resulting in osseous 


\section{ARTICLE SUMMARY}

Strengths and limitations of this study

- The study was performed prospectively in 62 consecutive MM patients.

- The two scan modalities were performed within 2 days of each other and were assessed uniformly by expert radiologists and nuclear medicine physicians in order to minimise possible bias.

- This is considered as the first study with head-to-head comparison between both modalities, and can therefore be used as a basis for larger studies.

- It was assumed that the MRI and the MIBI scan scoring scales were measuring the same disease properties. This, however, may have introduced an unknown degree of imprecision in our estimates.

- The studied group represents a heterogeneous group of myeloma patients. In theory, different treatments and lytic lesions may be detected by one method over the other due to the potential different sensitivity between both imaging modalities. However, this was not addressed by the current study and may require further investigation.

destruction. ${ }^{1-3}$ Most institutions utilise a skeletal survey in order to radiologically assess $\mathrm{MM}$ at diagnosis. ${ }^{3-5}$ Although skeletal survey was considered for many decades as a standard radiological technique in the diagnosis and staging of myeloma bone disease, it has a high false-negative rate of between $30 \%$ and $70 \%$ leading to underestimation of disease burden. ${ }^{6} 7$ MRI is currently used in several contexts in the monitoring of MM with high sensitivity compared with CT scan in detecting myeloma bone lesions. ${ }^{5-7}$ MRI is useful in identifying MM by its infiltration of normal fat within the bone marrow. ${ }^{5-8}$ Marrow infiltration seen on MRI can be used to assess the tumour burden present at diagnosis. ${ }^{7-9}$ In 2005, the Durie and Salmon staging system was refined to include whole body MRI (WB-MRI) where available. ${ }^{10} 11$

Sestamibi Technetium-99m MIBI bone marrow scanning (MIBI) accurately represents myeloma activity in the bone marrow with high sensitivity and specificity. ${ }^{12-14}$ There is also a linear correlation between bone marrow biopsy results and uptake on MIBI scan. ${ }^{12}$ However, there are no available data comparing MRI and MIBI scans in assessing disease activity and mortality in MM. Currently, MRI is the imaging modality of choice in staging MM disease according to the Durie-Salmon-PLUS staging system. ${ }^{10}$ Radionuclide imaging offers a potential alternative or supplement to radiographic detection and assessment of disease activity. ${ }^{11-17}$ There is scant evidence comparing MRI and MIBI bone marrow scans in assessing disease activity in $\mathrm{MM}$ in a cohort of this size. Therefore, there is a growing interest and recognition of the important role of MIBI bone marrow scan in imaging malignant tumours.

\section{Rationale and aims of the study}

Bone disease occurs in about $90 \%$ of MM patients. ${ }^{3}$ There are four available routine radio-nuclear imaging techniques for the diagnosis of bone disease in MM, which are plain x-ray, CT, MRI and bone scans. ${ }^{5} 1115$ Another technology employing positron emission tomography (PET) is considered mainly for research purposes and is not performed routinely for the diagnosis of myeloma. Plain radiographs can only demonstrate lytic bone disease when $30 \%$ or above of trabecular bone has been lost, which represents a late stage of the disease. ${ }^{15}{ }^{16}$ Furthermore, skeletal survey is not sensitive enough to monitor the responses to therapy.

Technetium-99m bone scanning without sestamibi is not appropriate for evaluating myeloma bone disease since bone scans reflect osteoblastic activity and thus, underestimates the extent of osteolytic lesions characteristic for myeloma bone disease. ${ }^{12-14}$ MIBI scanning has been investigated in myeloma patients because it is concentrated in myeloma tissue and thus, was found to be more sensitive than skeletal survey $(77 \%$ vs $45 \%)$ and was highly specific for staging myeloma patients. ${ }^{17}$

MRI has been proven in many studies to be more accurate than plain x-ray or CT scan in detecting myeloma bone lesions with a higher sensitivity. ${ }^{6-9}$ Therefore, MRI is considered to be a useful tool in the prediction of myeloma disease response; hence, new guidelines have integrated MRI as part of the staging of myeloma bone disease. ${ }^{10}{ }^{11}$ However, WB-MRI is a potential imaging method for high-resolution screening of the whole skeleton in patients with MM. ${ }^{8}$

There are little data available regarding this new technique especially in conjunction with other newly developed techniques like Sestamibi Technetium-99m in diagnosis and follow-up of MM or prediction of disease outcome. To date there is no head-to-head comparison between WB-MRI and MIBI scans in MM.

This study aims to determine whether: (1) In simultaneous WB-MRI and MIBI scans in the same patients, do both scans produce the same estimate of disease load and location (intermodality reliability), or does one of the scans suggests more extensive disease than the other. (2) Is there a greater association between the estimate of the extent of bone disease in one or other of the scans and the likelihood of death?

\section{SUBJECTS AND METHODS}

This study was conducted at the Launceston General Hospital, a tertiary referral centre for Northern Tasmania, Australia and was approved by the state-wide Human Ethics Committee of Tasmania. Informed consent was obtained from all participants in accordance with the Declaration of Helsinki. The study was registered prospectively at the Australia and New Zealand Clinical Trial Registry web site under registration No: ACTRN12609000761268 and in the NHS-UK website: http://www.nhs.uk/Conditions/MRI-scan/Pages/clinicaltrial-details.aspx? TrialId=ACTRN12609000761268.

There were 62 consecutive patients with a median age of 62 years (range 37-88) and a male-to-female ratio of 
$33: 29$ recruited at a single centre. Median duration of follow-up was 16.8 months (maximum 24 months), during which 13 of 62 patients succumbed. Most of the patients had IgG (35 patients) myeloma while 15 patients had IgA myeloma, four patients had smouldering myeloma and the remaining eight patients had light chain myeloma. The WHO criteria were used for the diagnosis of MM. ${ }^{18}$

A standard therapy with dexamethasone and thalidomide was applied in most of the cases (table 1). Second-line treatment was started after failing to achieve at least a minimal response with the first treatment defined by disease progression of $25 \%$ in serum $\mathrm{M}$ protein (at least $5 \mathrm{~g} / 1$ ) or urinary Bence Jones protein (at least $200 \mathrm{mg} /$ day), or $50 \%$ increase of serum free light chain level, or $10 \%$ increase of marrow plasmacytosis, or increase of the number of lytic lesions, or the presence of hypercalcaemia (corrected serum calcium $>2.65 \mathrm{mmol} / \mathrm{l}$ ), or finally in case of non-toleration to the first-line treatment. The salvage therapy include lenalidomide (12) or bortezomib (8), determined by disease stage and toleration of treatment. Ten patients underwent bone marrow transplantation.

\section{Diagnostic management}

Once enrolled in the trial, the participants underwent a MIBI scan and a WB-MRI. The vast majority of patients had both scans on the same day and all of them were performed within $48 \mathrm{~h}$ of each other with the MRI scan performed first. This was to avoid any possible effect of the Sestamibi Technetium-99m injection on the MRI scan results. The scans were interpreted by two independent consultant radiologists and nuclear medicine physicians with greater than 15 years of experience. The results were converted into a spreadsheet format where the body was represented as 61 bone compartments for statistical analysis. The same radiologists and nuclear medicine physicians were used to interpret all the scans

\section{Table 1 Patient characteristics}

\begin{tabular}{|c|c|}
\hline Total number of patient & 62 \\
\hline \multicolumn{2}{|l|}{ Sex } \\
\hline Male & 33 \\
\hline Female & 29 \\
\hline Median age & 62 years (range, $37-88$ ) \\
\hline$<60$ years & 16 \\
\hline$>60$ years & 46 \\
\hline Disease type & Number of patient \\
\hline $\lg G$ & 35 \\
\hline $\lg A$ & 15 \\
\hline Smouldering & 4 \\
\hline Free light chain disease/Bence Jones myeloma & 8 \\
\hline \multicolumn{2}{|l|}{ Free light chain } \\
\hline$\kappa$ & 32 \\
\hline$\lambda$ & 26 \\
\hline No light chain & 4 \\
\hline \multicolumn{2}{|l|}{ Clinical stage } \\
\hline 1 & 4 \\
\hline ॥ & 20 \\
\hline III & 38 \\
\hline Higher staging after MRI scan compared to skeletal survey & 14 \\
\hline Higher staging after MIBI scan compared to skeletal survey & 15 \\
\hline \multicolumn{2}{|l|}{ Lines of treatment } \\
\hline \multicolumn{2}{|l|}{ First-line treatment } \\
\hline Thalidomide/dexamethasone & $57 / 62$ \\
\hline Radiotherapy only & 2/62 \\
\hline Lenalidomide second-line therapy & $12 / 57$ \\
\hline Bortezomib second-line therapy & $8 / 57$ \\
\hline No treatment & $3 / 62$ \\
\hline Bone marrow transplantation salvage therapy & $10 / 57$ \\
\hline \multicolumn{2}{|l|}{ Cytogenetic risk stratification } \\
\hline Normal karyotype & 36 \\
\hline Complex cytogenetic with $>5$ abnormalities & 11 \\
\hline Complex cytogenetic including chromosome 13 abnormality & 4 \\
\hline Abnormal karyotype with less than 5 cytogenetic abnormalities & 5 \\
\hline Insufficient marrow cells or unable to grow bone marrow culture & 6 \\
\hline
\end{tabular}


using the same special format. Standardised protocols and assessments were used for both MRI and MIBI scans.

\section{MRI protocol}

The same machine was used for all the MRIs performedan Avanto 1.5T system from Siemens Medical Solutions. A similar protocol to Baur-Melnyk et $a l^{8}$ was employed with some modifications. The patients were placed head-first in the supine position. Head, neck, body-matrix and peripheral coils were utilised. For the examination of the spine, T1-weighted turbo spin-echo (TR/TE 584/10 for upper and 520/11 for lower, field of view $350 \mathrm{~mm}$ upper $380 \mathrm{~mm}$ lower, slice thickness $3 \mathrm{~mm}$ upper, $3.5 \mathrm{~mm}$ lower) and turbo spin echo short tau inversion recovery (STIR; TR/ TE/T1 5000/51/180, field of view $350 \mathrm{~mm}$ upper, $380 \mathrm{~mm}$ lower, slice thickness $3 \mathrm{~mm}$ upper $3.5 \mathrm{~mm}$ lower) were performed in the sagittal plane.

The base of skull and vertex were imaged in the axial plane with T1-weighted spin-echo (TR/TE 575/11, field of view $230 \mathrm{~mm}$, slice thickness $5 \mathrm{~mm}$ ) and turbo spin echo STIR (TR/TE/T1 6980/11/180). The sternum and posterior ribs were imaged in the coronal plane with T1-weighted turbo spin echo (TR/TE 596/7.8, field of view $480 \mathrm{~mm}$, thickness of slice $5 \mathrm{~mm}$ ) and turbo spin echo STIR (TR/TE/T1 7490/100/170). However, the ribs are too small for their internal marrow signal to be confidently interpreted, especially on T1 images, unless they are obviously destroyed or if there is a large lesion arising from the ribs. The pelvis was imaged in the coronal plane with T1-weighted turbo spin echo (TR/ TE 682/11, field of view $480 \mathrm{~mm}$ and slice thickness $5 \mathrm{~mm}$ ) and turbo spin echo STIR (TR/TE 4200/87/ 170). The lower limbs down to the mid-shin of the tibia were imaged in the coronal plane spin with T1-weighted turbo spin echo (TR/TE 504/11, field of view $480 \mathrm{~mm}$, slice thickness $5 \mathrm{~mm}$ ) and turbo spin echo STIR (TR/ TE/T1 3850/87/170). The mean time for each scan to occur was $50 \mathrm{~min}$.

\section{Sestamibi bone marrow scan protocol}

The same dose of MIBI-30 mCi (1110 MBq)—was used for each patient. Prior to intravenous injection, each patient was rested on the bed for at least $20 \mathrm{~min}$.

Imaging started at 5 min postinjection with a wide field of view $\gamma$-camera equipped with a low-energy highresolution collimator. The WB scan proceeded at $30 \mathrm{~cm} / \mathrm{min}$. When completed, lateral femora images were taken with each view, took approximately $2 \mathrm{~min}$ and, if required, a single positron emission CT was performed.

\section{Outcome measures}

The extent of bone MM as interpreted using the MRI scans was classified anatomically as focal or diffuse and then, according to intensity of the lesion, to high, intermediate and low. The extent of bone marrow MIBI uptake was scored accordingly. Morphology was classed as normal, diffuse, focal or micronodular. Severity was graded as low $(<20 \%$ infiltration $)$, intermediate (20-50\% infiltration) or high (>50\% infiltration), and the severity scale was applied only to the focal morphology. The severity and grade of positive diagnostic imaging reports were treated as a single rank order scale: $0=$ no lesions; $1=$ low-density focal; $2=$ intermediatedensity focal; $3=$ =high-density focal; $4=$ focal diffuse; $5=$ diffuse. The posterior ribs could not be imaged in the MRI scan, but the sternum could be imaged. Thus, the results for 25 ribcage bones in each patient (12 ribs by 2 sides plus sternum) were available for the MIBI and MRI-STIR scans, and one ribcage bone result (sternum only) was available for the MRI-T1 scan.

In a number of the result scale versus scan categories, no patient existed in that condition, or there was no death in a patient with that condition. In order to allow analysis of mortality data: (1) the no detection and lowintensity focal disease categories were combined and (2) the focal diffuse and diffuse categories were combined. The mortality status of the patients in January 2012 was determined using the Australian National Death Register to avoid distress to relatives caused by sending communications to patients who have died.

\section{Statistical analysis}

Concordance between MIBI and WB-MRI (either axial plane with T1 or turbo spin echo STIR) was tested by calculation of the kappa statistic for inter-rater agreement, expressed as observed and expected agreement (expected agreement is based on the random distribution of diagnostic results); $\kappa$ value (range -1.00 to 0 to 1.00 indicating high discordance to no concordance to full concordance, respectively); and $\mathrm{p}$ value. The number of positive results reported from the three scan modalities for each bone group (using the scan scale score for each scan as the outcome variable and the scan method as the predictor variable) were compared in the same patients at the same time using repeated-measures ordered logistic regression (a non-parametric equivalent of general linear modelling; with results expressed as OR with 95\% CIs and $p$ values). Adjustment for age, gender and time from diagnosis did not alter the OR estimates, so no adjusted results are reported for this analysis. Each group of bones was assessed separately, but the readings from all the bone compartments in each group were pooled. The association between time in months from the scan performance to death or censoring date of 1 January 2012 and the severity and grade of positive diagnostic results was compared using repeated-measures Cox proportional hazards regression (HR; 95\% CIs; $p$ values), adjusted for age and gender, time from diagnosis to imaging scans, clinical stage, number of lines of treatment, M-component and cytogenetic abnormalities. This was done using simultaneous interaction analysis with inclusion of each of the results of the three imaging modalities in the models, in order to determine which imaging modality most closely predicted mortality under the clinical management given to the 
patients. Results were corrected for multiple comparisons which were appropriate using the Holm method. All analyses were performed using Stata SE12.0 for Windows (StataCorp, College Station, Texas, USA).

\section{RESULTS}

We have analysed both scan results according to bone compartments. Furthermore, we have entered the results of MRI scan images in two different categories according to image type as MRI-T1 (axial plane with T1) or MRI-STIR (turbo spin echo STIR). In detecting skull lytic lesions, there were no significant differences between the scans; however, there was a tendency for MRI scan to detect more MM lesions than MIBI scan $(p=0.09)$. Nevertheless, for vertebral lytic disease (cervical, thoracic and lumbar), both MRI-T1 and MRI-STIR detected more disease than MIBI scan $(p<0.001)$. There was no statistical difference between both MRI techniques in detecting myeloma bone disease in the spine. In the pelvis, MRI-T1 detected significantly more disease than MIBI scan $(p=0.03)$ while there is no statistical difference between MRI-STIR and MIBI scan. In the long bones (femur, humerus, clavicle and scapula), MRI-T1 detected less disease than MIBI scan $(p<0.001)$, while there were no significant differences noted between both MRI-STIR and MIBI scans. In the ribcage, the MRI-T1 imaged the sternum but was not technically able to detect the myeloma involvement of the ribs, while the MIBI scan showed a significant ability in detecting lytic lesion of the ribs over MRI-STIR scan $(p<0.001)$.

Thirteen of the 62 patients died during the 24-month follow-up period, all from progression of MM. When the association between scan result and death rate secondary to disease progression (as measured by HR) was examined (figure 1), a clear step-wise increase in likelihood of death was seen for the MIBI scan for all bone compartments taken together, while the association was more irregular for the MRI scans (tables 2 and 3). This trend was seen in the combined all compartments analysis (MRI-T1 vs MIBI $\mathrm{p}=0.019$; MRI-STIR vs MIBI $\mathrm{p}=0.047$ ), the ribcage (MRI-T1 vs MIBI $\mathrm{p}=0.011$; MRI-STIR vs MIBI $\mathrm{p}=0.004)$, but was particularly strongly seen in the thoracic vertebrae (MRI-T1 vs MIBI $\mathrm{p}<0.001$; MRI-STIR vs MIBI $\mathrm{p}=0.001$ ). This pattern was less discernible in the skull, pelvis, long bones and cervical and lumbar vertebrae (figure 2). When adjusted for other prognostic indicators, in all bones combined, the mean MIBI scan result provides a better prediction of mortality than the mean result from the MRI scans (MRI_T1 vs MIBI HR for trend $0.70 ; 95 \%$ CI 0.53 to $0.92 ; \mathrm{p}=0.019$ : and MRI-STIR vs MIBI HR for trend 0.80 ; $95 \%$ CI 0.64 to 1.00 ; $\mathrm{p}=0.044$ ) when patients receive standard therapy for myeloma (table 3). If the combined thoracic vertebrae and ribcage bones are examined in isolation, the MIBI results provide a good prediction of mortality over 24 months (mean result per bone group HR for trend 2.58; 95\% CI 1.73 to $3.86 ; \mathrm{p}<0.001$ ), while the MRI scan results compared with MIBI had lower levels of prediction (MRI-T1 mean
A

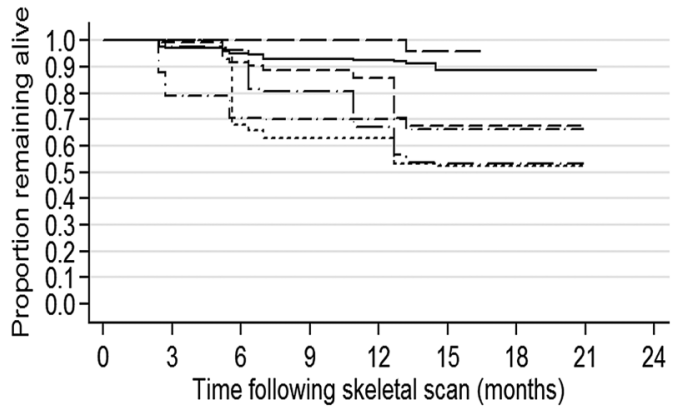

Number of bones at risk

\begin{tabular}{rrlllllllll} 
& No lesion & 1,318 & 1,283 & 1,250 & 1,223 & 1,222 & 998 & 526 & 100 & 0 \\
\hline$\ldots-$ & Focal (low density) & 169 & 168 & 155 & 150 & 145 & 106 & 71 & 0 & 0 \\
$\ldots \ldots . .$. & Focal (intermediate) & 103 & 103 & 70 & 65 & 65 & 54 & 32 & 0 & 0 \\
$-\ldots$ & Focal (high) & 201 & 196 & 194 & 162 & 135 & 104 & 35 & 0 & 0 \\
$-\ldots-$ & Diffuse focal & 70 & 70 & 70 & 70 & 70 & 67 & 0 & 0 & 0 \\
$\ldots \ldots$ & Diffuse & 268 & 212 & 189 & 188 & 188 & 151 & 59 & 0 & 0
\end{tabular}

B
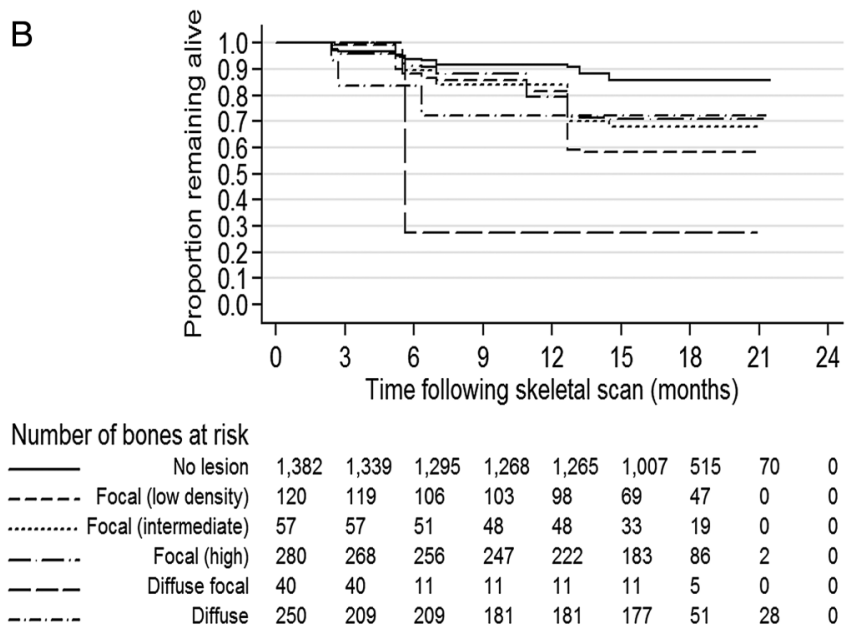

C

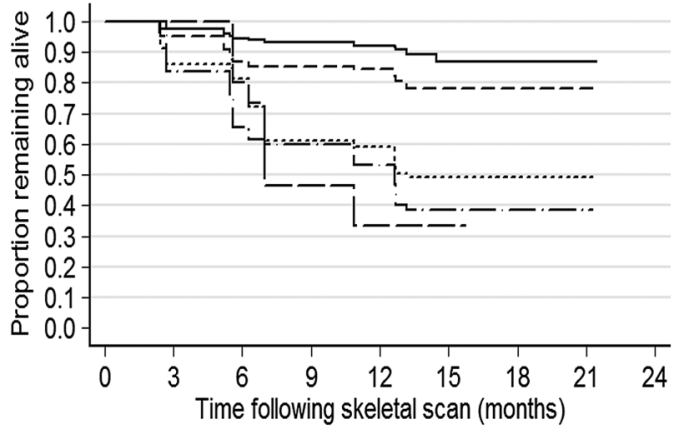

Number of bones at risk

\begin{tabular}{|c|c|c|c|c|c|c|c|c|c|}
\hline & No lesion & 1,511 & 1,473 & 1,429 & 1,411 & 1,394 & 1,123 & 603 & 75 \\
\hline-- & Focal (low density) & 301 & 287 & 262 & 257 & 255 & 217 & 60 & 4 \\
\hline ............ & Focal (intermediate) & 172 & 148 & 140 & 105 & 102 & 85 & 43 & 18 \\
\hline$-\cdot-\cdot$ & Focal (high) & 130 & 109 & 85 & 78 & 69 & 50 & 17 & 3 \\
\hline--- & Diffuse & 15 & 15 & 12 & 7 & 5 & 5 & 0 & 0 \\
\hline
\end{tabular}

Figure 1 Prediction of death due to disease progression by types of lesions in different imaging methods. In the 62 myeloma patients, the upper graph represents sestamibi bone marrow scan, middle graph; whole body MRI-T1 scan and the lower graph represents the STIR version of MRI scan.

result per bone group HR for trend 1.11; difference from MIBI 0.43; 95\% CI 0.27 to 0.67; $\mathrm{p}<0.001$ : MRI-STIR mean result per bone group HR for trend 1.14; difference from MIBI 0.44; $95 \%$ CI 0.30 to $0.66 ; \mathrm{p}<0.001)$. However, this bone grouping was not part of a prior hypothesis. Possible 
covariate associations with death rate were calculated for age (HR 3.88; 95\% CI 0.95-15.9; $\mathrm{p}=0.06$ ), gender (HR 2.69; $95 \%$ CI 0.41 to $17.6 ; \mathrm{p}=0.3$ ), time from diagnosis to scan prior to follow-up period (HR 3.38; 95\% CI 0.62 to 18.4; $\mathrm{p}=0.16$ ), presence of five or more cytogenetic abnormalities (HR 2.49; 95\% CI 0.77 to 8.02; $\mathrm{p}=0.13$ ) and lines of treatment (HR 1.44; 95\% CI 0.91 to 2.28; $\mathrm{p}=0.12$ ).

\section{Cost-effectiveness analysis}

There is no cost item for WB-MRI scan at present in the benefit scheme of Medicare Australia; however, based on approximate costs of skull MRI (\$403.20), pelvic MRI (\$403.20) and Spine MRI (\$358), the total costs would be about $\$ 1164.4$ while MIBI scan cost is $\$ 489.7$.
Nevertheless, a full body MRI scan may cost less than combining different body parts as it is less labour intensive and time consuming. At the time of analysis $\$ 1$ AUD is equal to $\$ 1 \mathrm{USD}$.

\section{DISCUSSION}

The true gold standard for myeloma disease detection would be bone marrow biopsy with histological, molecular biological and histochemical examination of the biopsy specimens in a laboratory where disease prognosis will be determined by the acquired genetic changes in the bone marrow cells and the resulting proliferation and location defects. This is obviously not feasible, since the relevant

Table 2 Estimates of concordance of grading* of results of MIBI, MRI and STIR scans in patients with multiple myeloma, and the relative frequency of different positive results

\begin{tabular}{|c|c|c|c|c|c|c|c|}
\hline & \multicolumn{4}{|l|}{ Concordancet } & \multicolumn{3}{|c|}{ Comparison of frequency } \\
\hline & Agreement (\%) & $\begin{array}{l}\text { Expected } \\
\text { agreement (\%) }\end{array}$ & kappa & p Value & OR & $95 \% \mathrm{Cl}$ & p Value \\
\hline \multicolumn{8}{|c|}{ Skull (N=61; \% positive 27.9\%) } \\
\hline MRI-T1 vs MIBI & 73.8 & 68.7 & 0.162 & 0.007 & 2.05 & (0.90 to 4.63$)$ & 0.09 \\
\hline MRI-STIR vs MIBI & 75.4 & 67.7 & 0.239 & 0.001 & 2.08 & (1.00 to 4.33$)$ & 0.10 \\
\hline MRI-STIR vs MRI-T1 & 85.3 & 61.8 & 0.614 & $<0.001$ & 1.02 & (0.61 to 1.70$)$ & 0.95 \\
\hline \multicolumn{8}{|c|}{ Cervical vertebrae ( $\mathrm{N}=434$; \% positive $34.3 \%$ ) } \\
\hline MRI-T1 vs MIBI & 65.7 & 62.8 & 0.076 & $<0.001$ & 7.26 & (5.11 to 10.3$)$ & $<0.001$ \\
\hline MRI-STIR vs MIBI & 61.8 & 59.9 & 0.046 & 0.004 & 8.13 & (5.66 to 11.7$)$ & $<0.001$ \\
\hline MRI-STIR vs MRI-T1 & 74.7 & 46.6 & 0.526 & $<0.001$ & 1.12 & (0.94 to 1.34$)$ & 0.21 \\
\hline \multicolumn{8}{|c|}{ Thoracic vertebrae ( $\mathrm{N}=744 ; \%$ positive $50.7 \%)$} \\
\hline MRI-T1 vs MIBI & 53.4 & 41.5 & 0.203 & $<0.001$ & 1.65 & $(1.43$ to 1.91$)$ & $<0.001$ \\
\hline MRI-STIR vs MIBI & 49.5 & 39.5 & 0.165 & $<0.001$ & 1.87 & (1.60 to 2.19$)$ & $<0.001$ \\
\hline MRI-STIR vs MRI-T1 & 71.5 & 39.8 & 0.527 & $<0.001$ & 1.13 & (0.98 to 1.31$)$ & 0.10 \\
\hline \multicolumn{8}{|c|}{ Lumbar vertebrae ( $\mathrm{N}=310$; \% positive $51.6 \%$ ) } \\
\hline MRI-T1 vs MIBI & 55.5 & 39.4 & 0.266 & $<0.001$ & 1.77 & (1.45 to 2.16$)$ & $<0.001$ \\
\hline MRI-STIR vs MIBI & 51.9 & 40.3 & 0.195 & $<0.001$ & 1.58 & (1.26 to 2.00$)$ & $<0.001$ \\
\hline MRI-STIR vs MRI-T1 & 76.8 & 38.0 & 0.626 & $<0.001$ & 0.90 & (0.75 to 1.08$)$ & 0.25 \\
\hline \multicolumn{8}{|c|}{ Pelvis ( $\mathrm{N}=183$; \% positive $60.7 \%$ ) } \\
\hline MRI-T1 vs MIBI & 48.6 & 32.0 & 0.245 & $<0.001$ & 1.37 & (1.06 to 1.78$)$ & 0.032 \\
\hline MRI-STIR vs MIBI & 53.0 & 36.1 & 0.265 & $<0.001$ & 0.85 & (0.66 to 1.10$)$ & 0.21 \\
\hline MRI-STIR vs MRI-T1 & 68.9 & 33.9 & 0.529 & $<0.001$ & 0.62 & (0.49 to 0.78$)$ & $<0.001$ \\
\hline \multicolumn{8}{|c|}{ Long bones (femur, humerus, scapular and clavicles) ( $\mathrm{N}=343$; \% positive $30.0 \%$ ) } \\
\hline MRI-T1 vs MIBI & 76.7 & 62.8 & 0.374 & $<0.001$ & 0.62 & (0.49 to 0.80$)$ & $<0.001$ \\
\hline MRI-STIR vs MIBI & 65.9 & 55.3 & 0.236 & $<0.001$ & 1.23 & (0.93 to 1.62$)$ & 0.15 \\
\hline MRI-STIR vs MRI-T1 & 78.4 & 61.3 & 0.443 & $<0.001$ & 1.97 & (1.49 to 2.60$)$ & $<0.001$ \\
\hline \multicolumn{8}{|c|}{ Ribcage§ (MRI vs MIBI and MRI vs STIR: N=54; \% positive $55.6 \%$ ) (STIR vs MIBI: N=1,159; \% positive $39.2 \%$ ) } \\
\hline MRI-T1 vs MIBI & 55.6 & 35.4 & 0.313 & $<0.001$ & 1.02 & (0.61 to 1.72$)$ & $>0.90$ \\
\hline MRI-STIR vs MIBI & 46.3 & 32.9 & 0.200 & 0.001 & 3.26 & (1.93 to 5.51$)$ & $<0.001$ \\
\hline MRI-STIR vs MRI-T1 & 70.4 & $39.6 \%$ & 0.510 & $<0.001$ & 1.29 & (0.79 to 2.11 ) & 0.62 \\
\hline
\end{tabular}

${ }^{*}$ Grading of diagnostic results of the bone scan, MRI and STIR scans were: 0 , no abnormality; 1 , focal low intensity; 2, focal intermediate; 3 , focal high; 4 , diffuse focal or micronodular; 5 , diffuse. $\mathrm{N}$ is number of patients multiplied by number of bones where duplicate simultaneous scans were available; \% positive is the percentage of bones where any of the scans were positive at any grade.

†Concordances between results of the MIBI scan, MRI and STIR scans, estimated by inter-rater kappa statistic (range 0 to 1.00 indicating high discordance to full concordance respectively; this statistic does not distinguish the direction of the disagreement; expected agreement is based on random distribution of diagnostic results).

$\ddagger$ Comparison of frequency and grade of positive detections of tumour masses by the MRI and STIR scans compared to that in the bone scans: estimated by unadjusted ordered logistic regression (assuming that higher grades indicate greater tumour masses), expressed as OR; $95 \%$ Cls; $p$ values) with $p$ values corrected for multiple comparisons by the Holm method.

$\S$ No result was recorded for any of the ribs in the MRI axial plane with T1 scans in any patient. The ribcage included the sternum, which was imaged by the T1 scans.

MIBI, Sestamibi Technetium-99m-bone marrow; STIR, short tau inversion recovery. 
Table 3 Disease progression risk with subsequent mortality in association with positive bone marrow scan (MIBI) or MRI results

\begin{tabular}{|c|c|c|c|c|c|c|}
\hline & \multirow[b]{2}{*}{ Result* } & \multicolumn{5}{|c|}{ Mean result in each bone groupt } \\
\hline & & Total & Died & HR‡ & $95 \% \mathrm{Cl}$ & p Value \\
\hline \multirow[t]{7}{*}{ MIBI } & 0 & 32 & 1 & 1.00 & & \\
\hline & 1 & 16 & 4 & 2.50 & (1.18 to 5.30$)$ & 0.05 \\
\hline & 2 & 11 & 6 & 4.17 & (1.60 to 10.9$)$ & 0.014 \\
\hline & 3 & 3 & 2 & 6.27 & (2.22 to 17.7$)$ & 0.003 \\
\hline & 4 & & & & & \\
\hline & 5 & & & & & \\
\hline & Absolute trend & & & 1.51 & (1.20 to 1.91$)$ & 0.001 \\
\hline \multicolumn{2}{|c|}{ Trend relative to $\mathrm{MIBI}$} & & & 1.00 & & \\
\hline \multirow[t]{7}{*}{ MRI-T1 } & 0 & 29 & 2 & 1.33 & (0.92 to 1.93$)$ & 0.13 \\
\hline & 1 & 10 & 3 & 2.20 & (0.29 to 16.8$)$ & $>0.90$ \\
\hline & 2 & 4 & 2 & 1.10 & (0.05 to 25.9$)$ & 0.83 \\
\hline & 3 & 10 & 4 & 2.48 & (0.33 to 18.6$)$ & $>0.90$ \\
\hline & 4 & 8 & 2 & 1.88 & (0.11 to 33.5$)$ & $>0.90$ \\
\hline & 5 & 1 & 0 & & & \\
\hline & Absolute trend & & & 1.06 & (0.83 to 1.35$)$ & 0.44 \\
\hline \multicolumn{2}{|c|}{ Trend relative to $\mathrm{MIBI}$} & & & 0.70 & (0.53 to 0.92$)$ & 0.019 \\
\hline \multirow[t]{7}{*}{ MRI-STIR } & 0 & 23 & 1 & 1.01 & (0.64 to 1.59$)$ & $>0.90$ \\
\hline & 1 & 16 & 3 & 1.61 & (0.18 to 14.7$)$ & 0.006 \\
\hline & 2 & 5 & 3 & 2.34 & (0.13 to 41.6$)$ & 0.13 \\
\hline & 3 & 7 & 2 & 4.37 & (0.46 to 41.7$)$ & 0.004 \\
\hline & 4 & 10 & 4 & 2.32 & (0.15 to 36.1$)$ & $<0.001$ \\
\hline & 5 & 1 & 0 & & & \\
\hline & Absolute trend & & & 1.20 & (1.01 to 1.44$)$ & 0.044 \\
\hline \multicolumn{2}{|c|}{ Trend relative to $\mathrm{MIBI}$} & & & 0.80 & (0.64 to 1.00$)$ & 0.047 \\
\hline
\end{tabular}

${ }^{*}$ Results of investigation: None, no lesion; 1 , focal (low density); 2, focal (intermediate); 3, focal (high); 4, diffuse focal; 5, diffuse.

$\dagger$ The mean result for each bone group was taken as the predictor of disease progression.

$\ddagger$ Association between result score of imaging modality: relative risk as HR ( $95 \% \mathrm{Cls}$; $p$ value) was estimated using repeated-measures Cox proportional hazards regression, adjusted for age, gender, time from diagnosis to imaging scans, clinical stage, number of lines of treatment, M-component and cytogenetic abnormalities; $p$ values corrected for multiple comparisons by the Holm method. Each result score value was first treated as a categorical variable, and then as a continuous variable to estimate the linear trends: the trend for each modality was expressed as an absolute effect, and then the trends of the two MRI modalities were expressed as relative effects compared with the MIBI trend.

MIBI, Sestamibi Technetium-99m-bone marrow; STIR, short tau inversion recovery.

bones may be inaccessible, and the biopsy process would be destructive and unacceptable to patients and treating physicians.

Here, the clinicians are forced to apply indirect scanning methods, which involve transmission or emission of various radiations (x-ray, CT, MRI or nuclear medicine scans). These depend not on the absolute properties of the abnormal cells that directly control the disease severity, but on the relative absorption/transmission of the radiation compared with the non-diseased tissue. Therefore, each method will have its own rate of false-positive and false-negative results. Nonetheless, because none of these modalities has been validated against the true measure of the disease, those rates are unknown. However, as an indirect measure of this gold standard, the disease progression experience of the patients is used as a proxy for disease severity.

There are a number of positive findings which have emerged from our study. As a direct comparison between both MIBI and WB-MRI scans in diagnosis of bone disease of MM, it seems that the MRI scan identifies more MM bone disease than the MIBI scan with more sensitivity in detecting myelomatous deposits in the spine and to a lesser extent in the pelvis and long bones. In the ribcage, MIBI scan detected more disease compared with MRI scan $(p<0.001)$, noting that the MRI scan of the ribcage is technically more difficult due to patient movement including breathing. Comparison between axial T1-weighted and STIR sequences shows that the former is more sensitive in detecting myelomatous disease in the vertebrae than STIR imaging. This is largely because the lesions are predominantly of intermediate and low signal at both T1-weighted and STIR imaging, and are better outlined and contrasted when marrow fat signal is preserved, as in T1-weighted imaging. The STIR sequence selectively suppresses the signal from fatty marrow in the spine, reducing lesion contrast and detection sensitivity (figure 3 ). Lesions that are of intermediate or low signal at T1-weighted MRI and high signal at STIR MRI are in the minority. Our analysis compares the results of the three scans (two interpretations of an MRI scan and an MIBI scan) for 
Figure 2 Association between result score of imaging modality: relative risk as $\mathrm{HR}(95 \% \mathrm{Cls}$; $p$ value) was estimated using Cox proportional hazards regression, adjusted for age, gender and time from diagnosis to imaging scans; $p$ values corrected for multiple comparisons by the Holm method. Each result score value was treated as a continuous variable to estimate the linear trends in mortality: the absolute trend for each modality was estimated (shown as symbol and error bars), and then the relative trends of the two MRI modalities were compared to the MIBI scan trend (shown as $p$ values only).

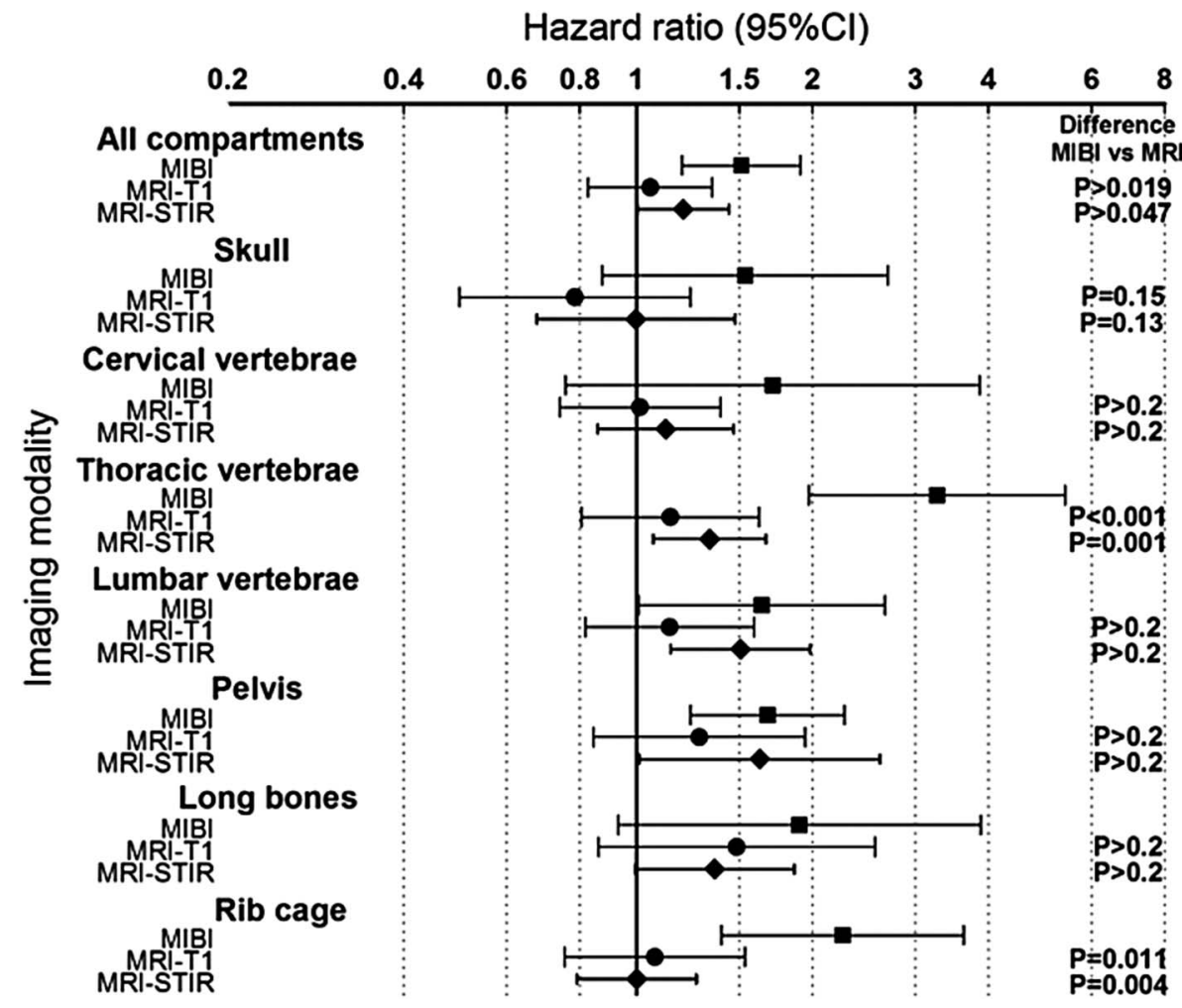

each patient, and then sums those comparisons across the entire patient group. Since each patient has the same age, gender and time from diagnosis for that internal comparison, those covariates will have no effect on the intrasubject comparison. Unless there is a systematic strong effect on the difference between the scans (which was not seen), the estimates of the difference across the group will not be changed. The pattern of prognostic prediction differs from the degree of identification of MM bone lesions suggesting that the nuclear medicine MIBI scan provides a better prediction of disease mortality than either of the MRI sequences (MRI-T1 vs MIBI $\mathrm{p}=0.19$; MRI-STIR vs MIBI $\mathrm{p}=0.047$ ). This may suggest that some MRI-detected lesions are of lesser significance than those imaged by the MIBI scan. This is intuitively unsurprising for at least two possible reasons: (1) not all lesions that are of intermediate or low signal on T1-weighted and STIR imaging represent myelomatous deposits; and (2) myeloma deposits that are clinically quiescent may appear morphologically similar to those that progress. It is worth noting that both MIBI scan and WB-MRI scans performed better than conventional skeletal plain x-ray survey as 15 patients and 14 patients, respectively, had a higher clinical staging according to both scans compared with routinely performed x-ray examination (table 1 ).

Several trials compared WB-MRI scan versus CT scan and showed superior results favouring the MRI scan. $^{7} 8{ }^{10}$ However, no correlation has been made in the prediction of overall disease outcome or death rate as our study suggests. On the other hand, one study showed that MIBI scan detects additional MM sites compared with skeletal survey in 38 of 56 studied patients $(68 \%)$. This is considered superior to FDG-PET scan that detects $50 \%$ of additional sites for MM over skeletal survey. $^{19}$

Our study has a few shortcomings. First, the two scales of assessment of severity of MM bone involvement (none, focal, focal-diffuse and diffuse) and signal density (low, intermediate and high, applied only to focal morphology) were assumed to be able to be converted into a single sequential scale, that is, positively associated with tumour mass in order to simplify the analysis. It was also assumed that the MRI and the MIBI scan scoring scales were measuring the same disease properties. This, however, may have introduced an unknown degree of imprecision in our estimates. Second, all the patients were receiving standard chemotherapy for myeloma, and tumour infiltration that can be detected by one technique may be influenced by certain therapy compared with a tumour that can be detected by the other imaging modality.

In summary, MIBI scan obtains an image of all important bone compartments in the body in one single examination, and is less time consuming and more comfortable for the patient than MRI. Although there is a mild-to-moderate degree of intermodality reliability between the different imaging techniques, the two MRI modalities appear to report higher grades of bone lesions than the MIBI scan. Moreover, our study suggests that mortality is better predicted by components of the MIBI scan, although this observation would need to be 


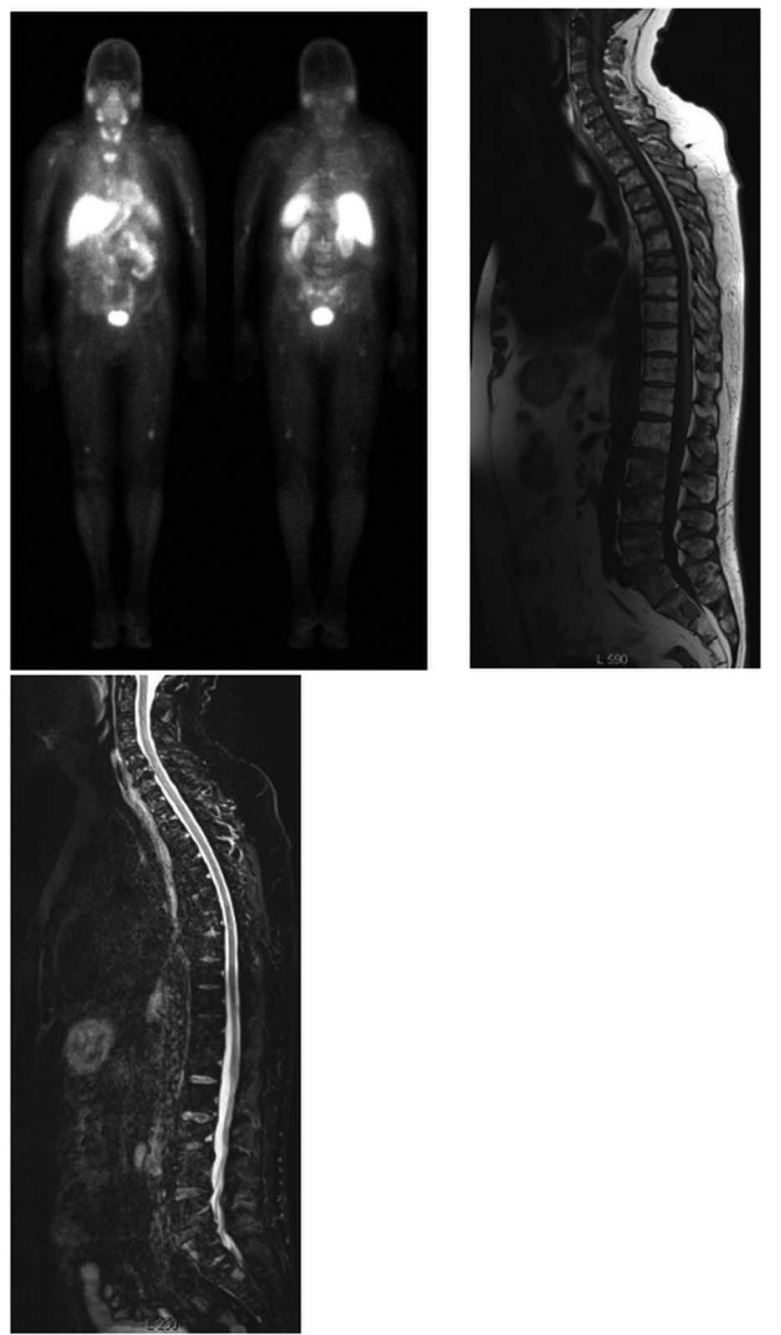

Figure 3 The image on the upper left is a Sestamibi Technetium-99m-bone marrow scan that demonstrates high-grade focal lesions in the sacrum plus L3, L4 and L5 vertebrae. Low-grade focal lesions are also seen in the T1 vertebrae, right scapula, right-sided sixth rib, bilateral femora and humeri. The upper right is a T1-weighted MRI of the spine that demonstrates high grade focal lesions from $L 2$ to L5 and in the sacrum in the same patient. Low-grade focal lesions are also demonstrated in the T4-T7 vertebrae while T7 shows an old compression fracture. The lower image is a short tau inversion recovery sequence (STIR) MRI of the spine that demonstrates heterogeneous signal from L2-L5, compatible with but not as obvious as the findings on the T1 image in the lumbar region of the same patient in our study cohort.

confirmed in other studies. The greater apparent lesion detection and poorer prognostic performance of MRI scans may occur either through imaging of malignant tissue that has been possibly affected by chemotherapy, or through imaging of tissue reactions that have little prognostic significance. Alternatively, the MRI scans may be more sensitive to early disease than MIBI scans and that early disease may manifest itself at a date beyond our short window of observation. Our data suggest that it is beneficial to employ the new technology with
WB-MRI and MIBI scans in prediction of myeloma disease progression as well as outcome. Further trials will be required to confirm the utility of these different imaging modalities.

\section{Author affiliations}

${ }^{1}$ Department of Medicine and Clinical Haematology, Launceston General Hospital, Launceston, Tasmania, Australia

${ }^{2}$ School of Human Life Sciences, University of Tasmania, Launceston, Tasmania, Australia

${ }^{3}$ Northern Nuclear Medicine Tasmania, Launceston, Tasmania, Australia ${ }^{4}$ Department of Radiology, Launceston General Hospital, Launceston, Tasmania, Australia

${ }^{5}$ Department of Haematology and Bone Marrow Transplantation, The Royal Adelaide Hospital, Adelaide, South Australia, Australia

This paper was presented at the Annual Meeting of the Haematology Society of Australia and New Zealand, Sydney, NSW, Australia in October 2011 and 2012.

Acknowledgements The authors acknowledge Mr Garth Faulkner, Business Manager of the Radiology Department at the Launceston General Hospital for his continuous support that enable the conduction of the study. The authors also wish to acknowledge that statistical support was funded by the Clifford Craig Medical Research Trust, Launceston, Tasmania, Australia.

Contributors AAK is the principal investigator of the study who organised and coordinated all aspects of the research including all steps of the manuscript preparation. He designed the study and recruited the patients. He has contributed in writing, reviewing, editing and approving the manuscript in its final form. AS, RH and LBT contributed to study design, analysis and interpretation of data and revised the manuscript in its final form. IKR performed all statistical analyses and interpreted the data and wrote the results section and revised the manuscript in its final form. JA and RD conducted the scans for the patients and helped in drafting the manuscript and writing the results. $\mathrm{RH}$, SR collected the clinical data, performed the literature search and wrote the introduction and drafted the article and finally approved the manuscript.

Funding This research received funding from the Clifford Craig Medical Research Trust, Launceston, Tasmania, Australia. Clifford Craig Medical Research Trust is a local community organisation in Tasmania, Australia that supports medical research by providing monetary funds without interference in the research design or outcome. Clifford Craig Medical Trust Fund has no influence in the study design; subject, methods, data collection, analysis, interpretation of data; writing the research report or the decision to submit the manuscript for publication. The grant was used to support the research assistants.

Competing interests None.

Patient consent Obtained.

Ethics approval This study is approved by the Tasmanian Human Ethics Committee, Australia and all participants have signed an informed consent form in accordance with the Declaration of Helsinki. The study was registered prospectively in the Australian and New Zealand Clinical Trials Registry at http://www.ANZCTR.org.au under No: ACTRN12609000761268 and in the NHS-UK website:http://www.nhs.uk/Conditions/MRI-scan/Pages/ clinical-trial-details.aspx?Trialld=ACTRN12609000761268.

Provenance and peer review Not commissioned; externally peer reviewed. Data sharing statement No additional data are available.

\section{REFERENCES}

1. Kyle RA, Gertz MA, Witzig TE, et al. Review of 1027 patients with newly diagnosed multiple myeloma. Mayo Clin Proc 2003;78:21-33.

2. Rajkumar SV, Kyle RA. Multiple myeloma-diagnosis and treatment. Mayo Clin Proc 2005;80:1371-82.

3. Roodman GD. Pathogenesis of myeloma bone disease. Blood Cells Mol Dis 2004;32:290-2. 
4. Parkin DM, Bray F, Ferlay J, et al. Global cancer statistics, 2002. CA Cancer J Clin 2005;55:74-108

5. D'Sa S, Abildgaard N, Tighe J, et al. Guidelines for the use of imaging in the management of myeloma. $\mathrm{Br} J$ Haematol 2007;137:49-63.

6. Lütje S, de Rooy JW, Croockewit $\mathrm{S}$, et al. Role of radiography, MRI and FDG-PET/CT in diagnosing, staging and therapeutical evaluation of patients with multiple myeloma. Ann Hematol 2009;88:1161-8.

7. Delorme S, Baur-Melnyk A. Imaging in multiple myeloma. Recent Results Cancer Res 2011;183:133-47.

8. Baur-Melnyk A, Buhmann S, Becker C, et al. Whole-body MR versus whole-body MDCT for staging of multiple myeloma. AJR Am $J$ Roentgenol 2008;190:1097-104.

9. Hanrahan CJ, Christensen CR, Crim JR. Current concepts in the evaluation of multiple myeloma with MR imaging and FDG PET/CT. Radiographics 2010;30:127-42.

10. Durie BG, Kyle RA, Belch A, et al. Myeloma management guidelines: a consensus report from the Scientific Advisors of the International Myeloma Foundation. Hematol J 2003;4:379-98.

11. Dimopoulos M, Terpos E, Comenzo RL, et al. International myeloma working group consensus statement and guidelines regarding the current role of imaging techniques in the diagnosis and monitoring of multiple Myeloma. Leukemia 2009;23:1545-56.

12. Balleari $\mathrm{E}$, Villa $\mathrm{G}$, Garrè $\mathrm{S}$, et al. Technitium-99 m-sestaMIBI in multiple myeloma and related gammopathies: a useful tool in investigation and follow-up of myeloma bone disease. Haematologica 2001;86:78-84.

13. Fonti R, Salvatore B, Quarantelli M, et al. 18F-FDG PET/CT, 99mTc-MIBI, and MRI in evaluation of patients with multiple myeloma. J Nucl Med 2008;49:195-200.

14. El-Shirbiny AM, Yeung $\mathrm{H}$, Imbriaco $\mathrm{M}$, et al. Technetium-99m-MIB versus fluorine-18-FDG in diffuse multiple myeloma. J Nucl Med 1997;38:1208-10.

15. Roodman GD. Skeletal imaging and management of bone disease. Hematol Am Soc Hematol Educ Program 2008:313-19. doi:10.1182/ asheducation-2008.1.313

16. Roodman GD. Pathogenesis of myeloma bone disease. Leukemia 2009;23:435-41.

17. Mele A, Offidani M, Visani G, et al. Technetium-99m sestamibi scintigraphy is sensitive and specific for the staging and the follow-up of patients with multiple myeloma: a multicentre study on 397 scans. Br J Haematol 2007;136:729-35.

18. McKenna RW, Kyle RA, Kuehl WM. World Health Organization classification of tumours of haematopoietic and lymphoid tissues. In: Swerdlow SH, Campo E, Harris NL, Jaffe ES, Pileri SA, Stein H, Thiele J, Vardiman JW, eds. 3rd ed. IARC Press: Lyon, 2008;200-13.

19. Mileshkin L, Blum R, Seymour JF, et al. A comparison of fluorine-18 fluoro-deoxyglucose PET and technetium-99m sestamibi in assessing patients with multiple myeloma. Eur J Haemato 2004;72:32-7. 